\title{
Electromechanical Response of Conductive Porous Structure
}

\author{
Hye-Mi So, ${ }^{1}$ Cheolmin Park, ${ }^{2}$ and Won Seok Chang ${ }^{1,2}$ \\ ${ }^{1}$ Department of Nanomechanics, Korea Institute of Machinery and Materials, Daejeon 305-343, Republic of Korea \\ ${ }^{2}$ Department of Nanomechatronics, Korea University of Science and Technology, Daejeon 305-333, Republic of Korea \\ Correspondence should be addressed to Won Seok Chang; paul@kimm.re.kr
}

Received 26 September 2014; Revised 9 December 2014; Accepted 9 December 2014

Academic Editor: Christian Brosseau

Copyright (C) 2015 Hye-Mi So et al. This is an open access article distributed under the Creative Commons Attribution License, which permits unrestricted use, distribution, and reproduction in any medium, provided the original work is properly cited.

\begin{abstract}
Porous conductors with large surface-volume ratios have been applied to a variety of fields, including absorbents, flexible heaters, and electrodes for supercapacitors. In this study, we implemented sensitive pressure sensors using the mechanical and electrical characteristics of conductive porous structures manufactured by immersing sponges into a carbon nanotube solution and then measured the change in resistance. When pressure was applied to conductive sponges, carbon nanotubes were attached to each other and the resistance was reduced by up to $20 \%$. The carbon nanotube sponges, which were soft and had superior elasticity, were quickly stabilized without any changes taking place in their shape, and they showed consistent change in resistance during experiments of repetitive pressure. The pressure devices based on conductive porous sponges were connected to single-walled carbon nanotube field effect transistors (SWCNT-FETs) and changes in their characteristics were investigated according to external pressure.
\end{abstract}

\section{Introduction}

Conductive foam-like structures have attracted considerable attention as electrodes for supercapacitors, flexible heaters, and catalytic electrodes due to their high porosity, flexibility, and robustness under mechanical stress [1-5]. These structures have also demonstrated excellent electrical, mechanical, and chemical properties in performance. However, use of the foam-like structures has been limited because it is very difficult to synthesize porous structures with a high level of flexibility, robustness, and conductivity. In recent years, nanocomposites have been fabricated by introducing nanomaterials such as metal nanoparticles in the foam or by stacking carbon nanotubes or hydrothermal reduction of grapheme oxide dispersion with pyrrole polymer [6-8]. Carbon-based materials have much potential as sponge-like structures with high porosity, flexibility, and large deformability $[7,8]$. However, these nanocomposite foams tend to collapse or distort under mechanical stress due to their relatively poor compressibility and elasticity. On the other hand, regular polymer foams have many advantageous properties. Their density is low, so they are light and they are flexible and soft. For these properties, they are widely used in many application areas, such as disposable packaging, cushioning, and insulating material. The properties can also be tuned to specific applications by varying the density of the foam or by adding functional nanomaterial. We explored the porous and elastic properties of polymer foams in pressure sensors. In polymer foams, well-dispersed carbon nanotubes were randomly overlapped onto each other in the polymer scaffold.

Recently, many remarkable studies on flexible pressure sensors using flexible elements have been reported [9-13]. Schwartz et al. reported the flexible pressure sensitive organic thin film transistor with microstructured polydimethylsiloxane (PDMS) dielectric [9]. In most of these studies, pressure sensitive rubbers (PSRs) were utilized as sensing elements in flexible pressure sensors [10-13]. Arrays of sensors, in which conductive rubber films are attached to organic transistors, have the advantage that they can process complex signals by implementing active devices without interfering with adjacent devices $[10,11]$. Although PSR is flexible and pressure sensitive, it has the following disadvantages: it does not have the same characteristics under the same pressure due to contact resistance between polymers and electrodes; it has a low uniformity of film when it is implemented on a large area; and it has limitations in response time due to its large 


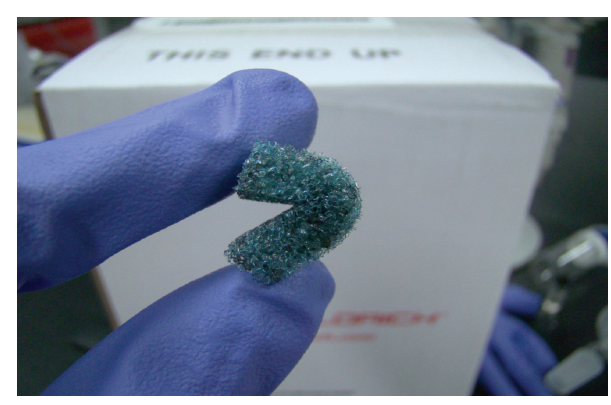

(a)

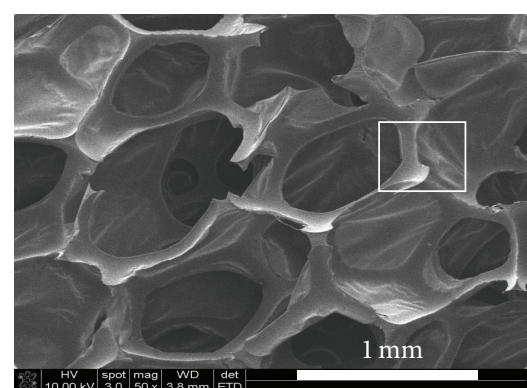

(b)

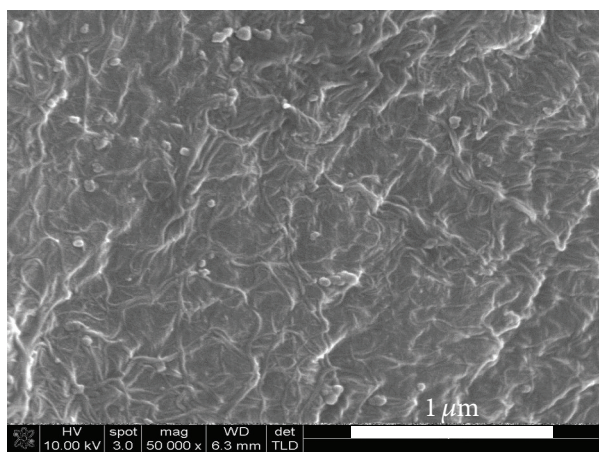

(c)

FIGURE 1: (a) Image of CNT sponge. (b) SEM image of CNT sponge. (c) SEM image of the region outlined in part (b). The scale bar is $1 \mu \mathrm{m}$.

hysteresis effect [11-13]. More improved pressure sensing materials that have superior elasticity and deformability to rubber type film are required. A soft and porous structure such as sponge is ideal for flexible sensing elements, and it also can be easily adapted to any three-dimensional object.

Here, we present a pressure detection device using conductive sponge that exhibits remarkable elasticity and restoration depending on pressure. The conductive sponges were prepared by an extremely simple "dipping and drying" process using single-walled carbon nanotube (SWCNT) solution. The pressure response of the conductive sponge was tested by measuring resistance change. Additionally, the electrical characteristics of a single-walled carbon nanotube field effect transistor (SWCNT-FET) combined with the sponge sensor depending on pressure were investigated.

\section{Experimental Section}

2.1. Preparation of Conductive Sponge. Sponges with porous structures that have excellent elasticity and restoring force were made conductive to implement resistant type pressure devices as follows: (1) sponges were immersed for 1 hour in a well-distributed SWCNT solution (TopNanosys Inc.) and then dried for 1 hour in an oven at $120^{\circ} \mathrm{C}$; (2) they were washed with DI water to remove surfactants in the solution and uncombined CNTs remained in them and were then dried. The conductivity of the sponge by different concentration of CNT solution can be easily adjusted.
2.2. Fabrication of SWCNT-FET. Semiconducting singlewalled carbon nanotubes $(10 \mu \mathrm{g} / \mathrm{mL}, 99 \% \mathrm{~s}-\mathrm{SWCNTs}$, NanoIntegris Inc.) were dispersed onto $\mathrm{SiO}_{2} / \mathrm{Si}$ substrate through the spray method. Repeated spraying and washing were carried out to remove the surfactants in SWCNTs distributed in water. Then the source and drain electrodes $(\mathrm{Cr} / \mathrm{Au})$ were formed by photolithography and thermal evaporation. The heavily doped Si substrate was used as the back gate electrode. SWCNTs that were randomly distributed on substrates were removed by $\mathrm{O}_{2}$ plasma treatment $\left(100 \mathrm{~W}, 300\right.$ mTorr, $\mathrm{O}_{2}$ $60 \mathrm{sccm}, 10 \mathrm{sec}$ ) except in the channel regions.

2.3. Pressure Response Characterization. In order to measure the resistance of the sponge, electrical wires were connected to the top and bottom of conductive sponges. Change in the resistance of the conductive sponge was measured with respect to pressure using a strain gauge-type load cell, and the electrical properties of the transistor were measured with a semiconductor parameter analyzer (Agilent E5262A). Moreover, pressure response of the SWCNT-FET was measured after combining the sponge pressure device and the SWCNTFET.

\section{Results and Discussion}

Conductive sponges with porous structures that have excellent elasticity and restoring force were made by immersing sponges into SWCNT solutions. Dried conductive sponges kept their original shape and characteristics even though they 


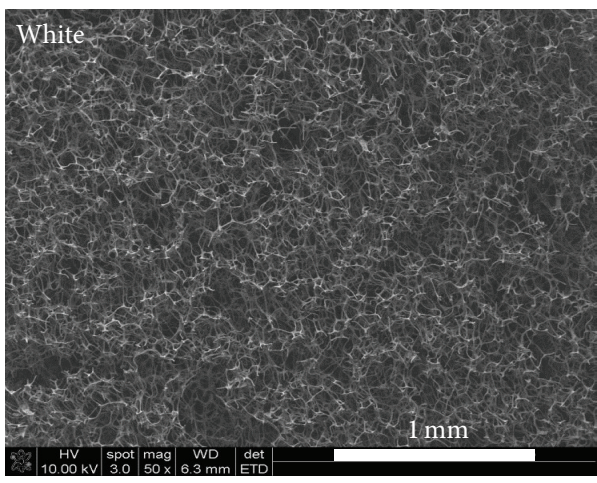

(a)

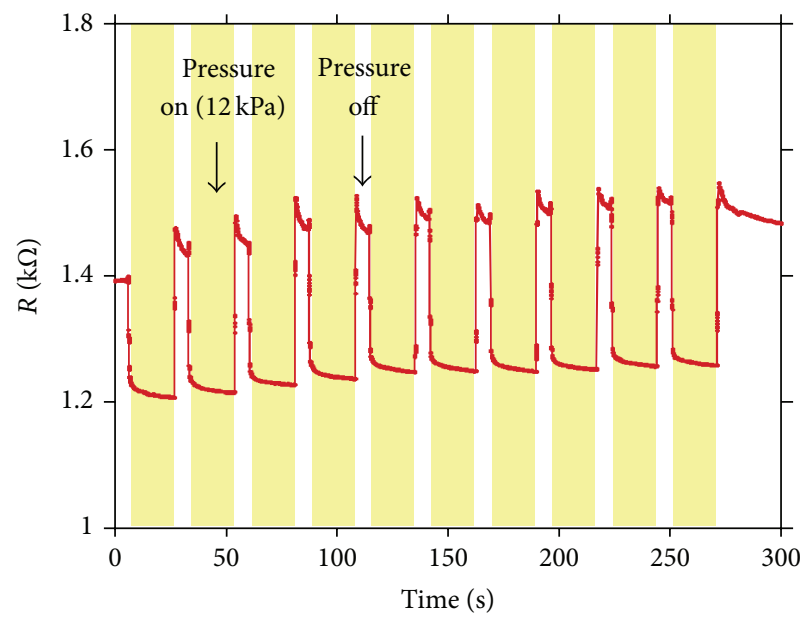

(c)

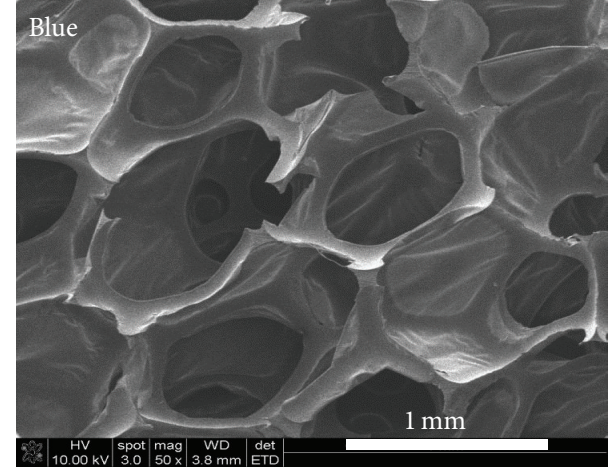

(b)

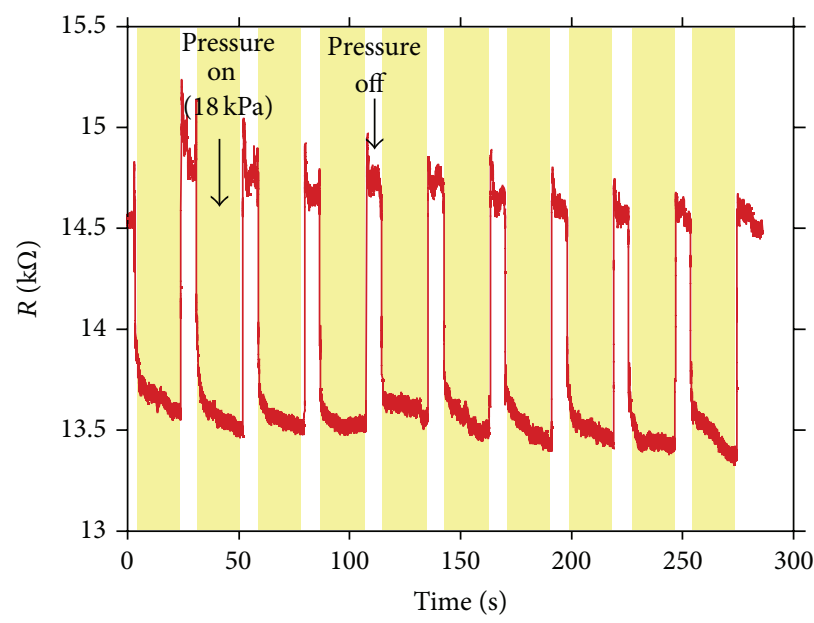

(d)

Figure 2: SEM images of (a) white and (b) blue sponge. (c, d) Pressure response of white sponge at $12 \mathrm{kPa}$ and blue sponge at $18 \mathrm{kPa}$, respectively.

were coated with SWCNTs. Many previous studies reported that carbon nanotubes and polymers were strongly combined by van der Waals interaction $[14,15]$. We found that SWCNTs were closely coated on the bones of sponges through a simple process of immersing them in the SWCNT solution as shown in Figure 1. Strongly combined carbon nanotubes were not separated after washing and their conductivity was maintained.

Two kinds of sponges with different bone thicknesses and pore diameters as shown in Figure 2 were used in this study to determine the pressure response according to the kinds of sponges. For the two kinds of sponges made under the same conditions, the average surface resistance of the blue sponges (polyethylene sponge) with relatively thick bones of approximately $200 \mu \mathrm{m}$ and large pore size $(\sim 500 \mu \mathrm{m})$ was $30 \mathrm{k} \Omega / \mathrm{sq}$ and that of white sponges (magic block) with bones thickness of $10 \mu \mathrm{m}$ and high density was $3.67 \mathrm{k} \Omega / \mathrm{sq}$. This indicates that the surface resistance decreased as the density of the bones, which are current paths, increased. Sponges were connected to electrodes to investigate pressure responses and then changes in the resistance were measured according to external pressure. Figures 2(c) and 2(d) show the changes in the resistance of each sponge. The resistance of white sponges decreased by $20 \%$ while that of blue sponges decreased by $7 \%$ at $12 \mathrm{kPa}$ and $18 \mathrm{kPa}$, respectively. Despite the different stress, the resistance tended to decrease when pressure was applied in both cases. The reason is supposed to be due to increases in the electrical path by the overlapped nanotubes when pressure was applied. We confirmed that resistance was clearly changed by pressure while changes in the resistance of the sponges were not larger than pressure sensitive rubber [16]. Even though the resistance change of the white sponge was larger in spite of the low pressure than the blue one, the white sponge was quite brittle. However, the blue sponge has a suitable resistance range for detecting pressure and is more rigid than white sponge. For this reason, the blue sponge was used in the SWCNT-FET combined pressure sensitive device.

The s-SWCNTs with a length of no more than $1 \mu \mathrm{m}$ and a diameter of $1.8 \mathrm{~nm}$ were uniformly networked with a density of more than 5 CNTs per unit area between the source and drain electrode. The channel length and width of the devices we fabricated are $8 \mu \mathrm{m}$ and $1000 \mu \mathrm{m}$, respectively. The sourcedrain current $\left(I_{\mathrm{ds}}\right)$ modulation characteristics of SWCNTFET as a function of bias voltage $\left(V_{\mathrm{ds}}\right)$ and gate voltage $\left(V_{g}\right)$ are shown in Figure 3. The gate voltages varied from -10 


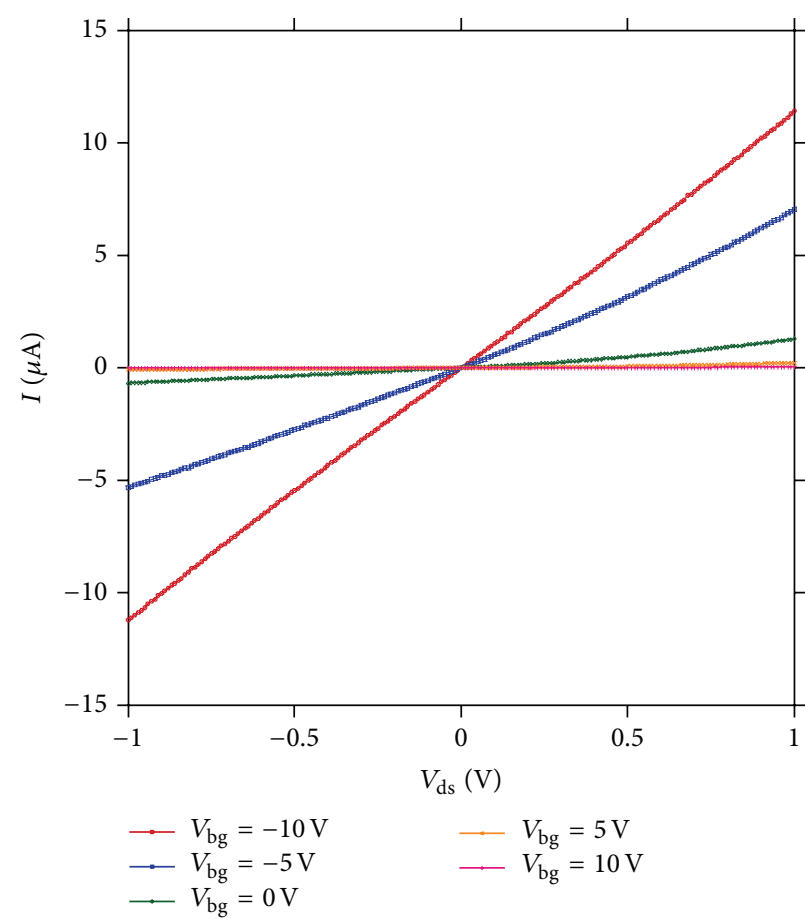

(a)

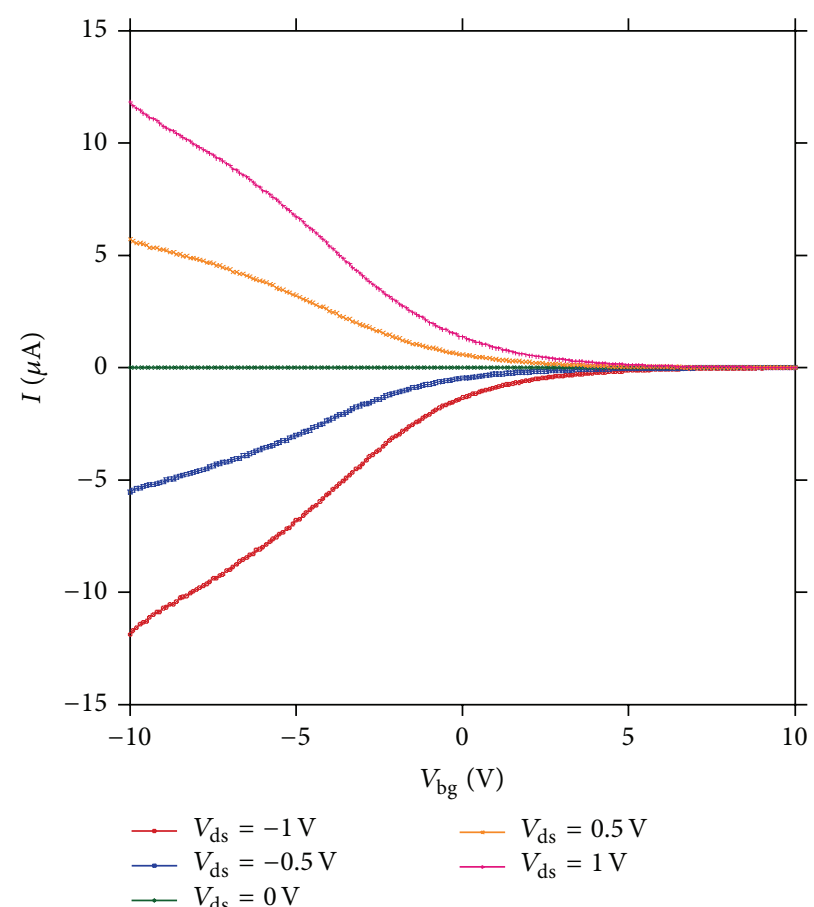

(b)

Figure 3: Electrical properties of the SWCNT-FET on $\mathrm{SiO}_{2} / \mathrm{Si}$ substrate. (a) Gate dependent output $\left(I_{\mathrm{ds}}-V_{\mathrm{ds}}\right)$ characteristics of the device. A gate voltage between $-10 \mathrm{~V}$ and $10 \mathrm{~V}$ was applied in $5 \mathrm{~V}$ steps. (b) Transfer curves of the device.

to $+10 \mathrm{~V}$. At the same $V_{\mathrm{ds}}$, lower $I_{\mathrm{ds}}$ were observed as the gate voltage increased from negative to positive voltages. The SWCNT device exhibits the typical $p$-type characteristic and on/off current ratio of $10^{3}$ as shown in Figure 3(b).

Because changes in the resistance of sponge itself by pressure are not larger than that of typical pressure sensitive rubber, we have integrated the conducting sponge sensor and SWCNT-FETs which can be expected to amplify the signal by FETs. Therefore, in order to maximize the effect, the blue sponge pressure device was directly put on the drain electrodes of the FET. Thus, the sponges play the role of electrodes as well as pressure devices. When the sponge pressure devices were placed on the drain electrodes of SWCNTFETs, the remaining parts except for drain electrodes were protected by PMMA film to prevent short circuits with the source and channel material as shown in Figure 4(a). Figure 4(b) shows the pressure response of the combined device, sponge pressure device, and SWCNT-FET, at different external pressures. The applied pressure induces a change in the resistance of the sponge, and then that transfers into a change in the drain current. When the drain voltage was fixed to $1 \mathrm{~V}$, the drain current increased as pressure increased. The reason is supposed to be due to the fact that the contact area between the conductive frame of the sponge pressure device and drain electrode increases as pressure increases. However, above a certain pressure, the amplitude of increase in the current significantly decreased and then it became saturated as shown in Figure 4(c). It is expected that the contact area of sponge pressure device and drain electrode is invariable over a specific pressure.

To consider effect of the contact area when the sponge contacted the electrode, the pressure dependence was tested as a control. Pressure sensitivity improved when the sponge connected with the SWCNT-FET. Even though the resistance change ratio of the sponge itself was low, a high response on pressure was obtained by connection with the FET as well as controlling of contact area. We attribute this difference to the amplification properties of the transistor and expect significant improvements in pressure response for sponges with the appropriate resistance range.

\section{Conclusions}

In summary, we demonstrated a conductive sponge pressure device with high sensitivity and relatively simple fabrication. The sponge structure was made conductive through a simple process in which a conventional sponge was immersed into a SWCNT solution and then dried. The pressure response depended on the thickness of the bones and the diameter of pores in the sponges and a reduction in resistance of up to $20 \%$ was confirmed. We also observed a much increased drain current at low pressure when a sponge pressure device and a SWCNT-FET were integrated. The drain current significantly increased by virtue of the effects of an instantaneous contact area and amplification of the transistor; the current was saturated to a constant value 

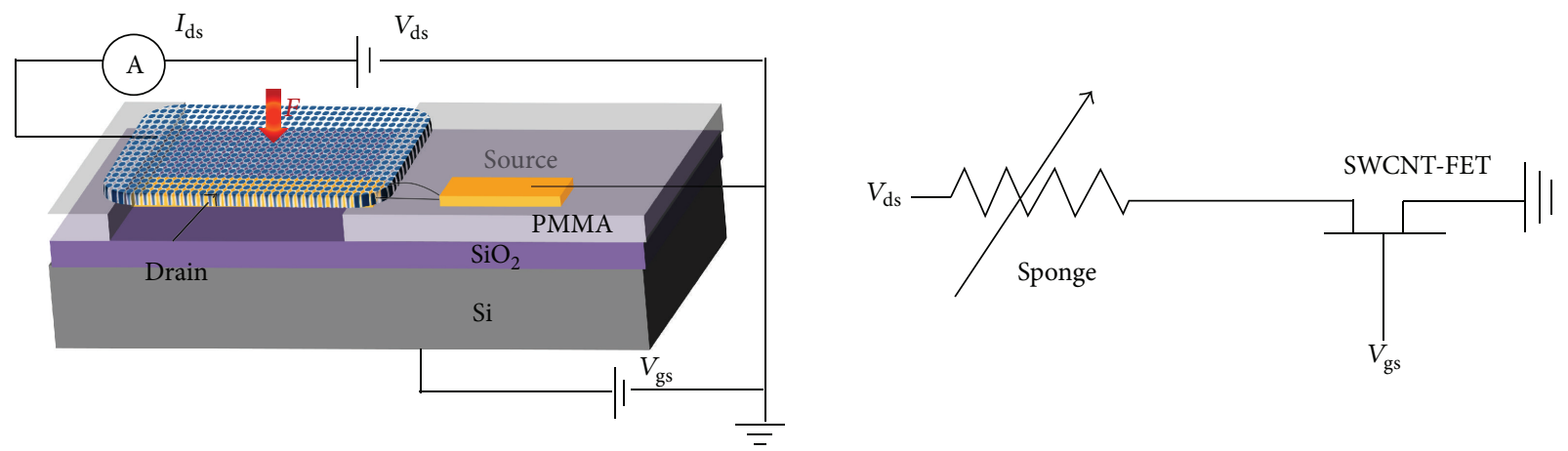

(a)
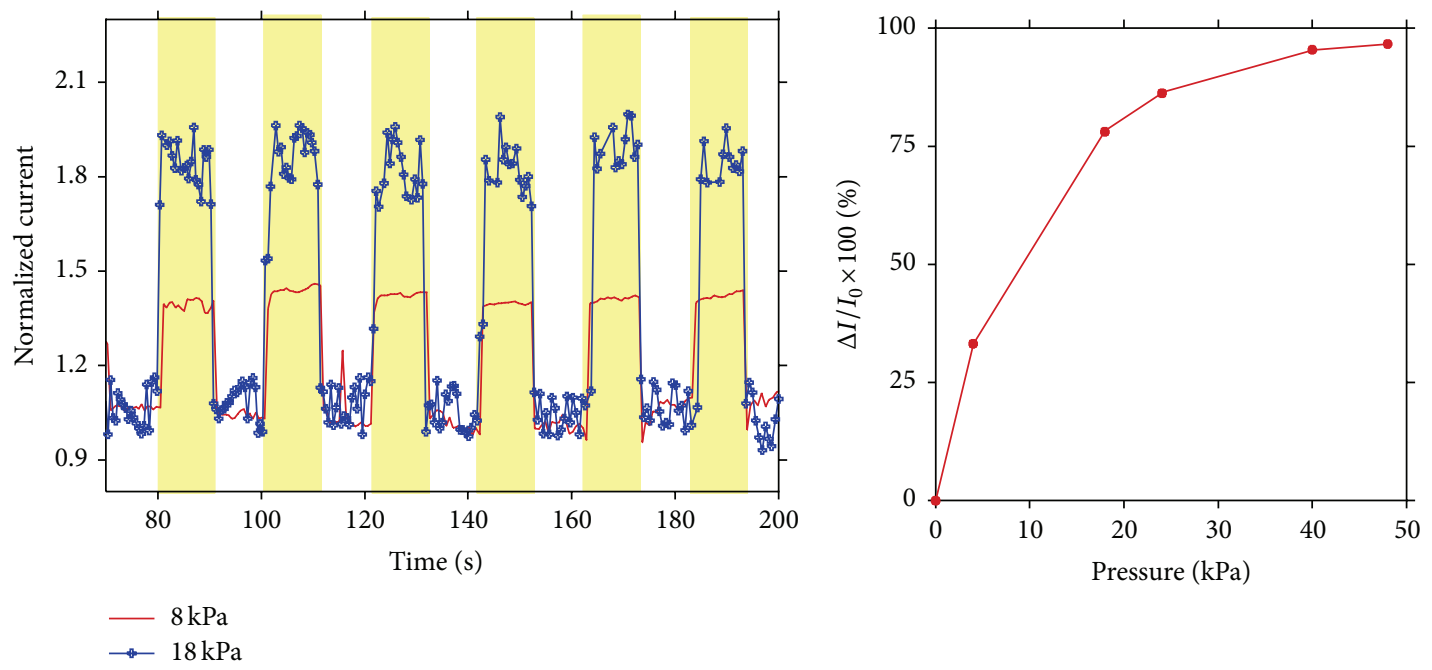

(b)

(c)

FIGURE 4: (a) Schematic diagram of the integrated system of a sponge pressure sensor and SWCNT-FET. (b) Pressure response of the drain current at $8 \mathrm{kPa}$ and $18 \mathrm{kPa}$, respectively. (c) Increase of the drain current as a function of pressure at constant voltage $\left(V_{\mathrm{ds}}=1 \mathrm{~V}\right.$ and $V_{\mathrm{gs}}=$ $-8 \mathrm{~V}$ ) when sponge was connected with FET.

beyond specific pressure. It is possible to implement arrays of pressure sensors that can process on-board signals by adjusting the contact area of sponges and the transistor's drain electrode. Furthermore, by introducing a porous structure, a desired resistance range appropriate to active materials as well as convenient deformation is possible.

\section{Conflict of Interests}

The authors declare that there is no conflict of interests regarding the publication of this paper.

\section{Acknowledgments}

This work was supported by the Center for Advanced Soft-Electronics as Global Frontier Project (CASE2013M3A6A5073183) and a grant-in-aid for Nanomaterial Technology Development Program (Green Nanotechnology Development Program) through the National Research Foundation of Korea (NRF) funded by the Ministry of Science, ICT and Future Planning.

\section{References}

[1] D. N. Futaba, K. Hata, T. Yamada et al., "Shape-engineerable and highly densely packed single-walled carbon nanotubes and their application as super-capacitor electrodes," Nature Materials, vol. 5, no. 12, pp. 987-994, 2006.

[2] X. Gui, J. Wei, K. Wang et al., "Carbon nanotube sponges," Advanced Materials, vol. 22, no. 5, pp. 617-621, 2010.

[3] J. E. Trancik, S. C. Barton, and J. Hone, "Transparent and catalytic carbon nanotube films," Nano Letters, vol. 8, no. 4, pp. 982-987, 2008.

[4] W. Chen, R. B. Rakhi, L. Hu, X. Xie, Y. Cui, and H. N. Alshareef, "High-performance nanostructured supercapacitors on a sponge," Nano Letters, vol. 11, no. 12, pp. 5165-5172, 2011.

[5] A. J. Svagan, M. A. S. A. Samir, and L. A. Berglund, "Biomimetic foams of high mechanical performance based on nanostructured cell walls reinforced by native cellulose nanofibrils," Advanced Materials, vol. 20, no. 7, pp. 1263-1269, 2008.

[6] E. Muñoz, M. de Val, M. L. Ruiz-González et al., "Gold/carbon nanocomposite foam," Chemical Physics Letters, vol. 420, no. 13, pp. 86-89, 2006.

[7] Z. Zeng, X. Gui, Z. Lin et al., "Carbon nanotube sponge-array tandem composites with extended energy absorption range," Advanced Materials, vol. 25, no. 8, pp. 1185-1191, 2013. 
[8] Y. Zhao, J. Liu, Y. Hu et al., "Highly compression-tolerant supercapacitor based on polypyrrole-mediated graphene foam electrodes," Advanced Materials, vol. 25, no. 4, pp. 591-595, 2013.

[9] G. Schwartz, B. C.-K. Tee, J. Mei et al., "Flexible polymer transistors with high pressure sensitivity for application in electronic skin and health monitoring," Nature Communications, vol. 4, article 1861, 2013.

[10] T. Someya, Y. Kato, T. Sekitani et al., "Conformable, flexible, large-area networks of pressure and thermal sensors with organic transistor active matrixes," Proceedings of the National Academy of Sciences of the United States of America, vol. 102, no. 35, pp. 12321-12325, 2005.

[11] K. Takei, T. Takahashi, J. C. Ho et al., "Nanowire active-matrix circuitry for low-voltage macroscale artificial skin," Nature Materials, vol. 9, no. 10, pp. 821-826, 2010.

[12] T. Sekitani, U. Zschieschang, H. Klauk, and T. Someya, "Flexible organic transistors and circuits with extreme bending stability," Nature Materials, vol. 9, no. 12, pp. 1015-1022, 2010.

[13] T. Someya, T. Sekitani, S. Iba, Y. Kato, H. Kawaguchi, and T. Sakurai, "A large-area, flexible pressure sensor matrix with organic field-effect transistors for artificial skin applications," Proceedings of the National Academy of Sciences of the United States of America, vol. 101, no. 27, pp. 9966-9970, 2004.

[14] V. L. Pushparaj, M. M. Shaijumon, A. Kumar et al., "Flexible energy storage devices based on nanocomposite paper," Proceedings of the National Academy of Sciences of the United States of America, vol. 104, no. 34, pp. 13574-13577, 2007.

[15] S. Yun, S.-D. Jang, G.-Y. Yun, and J.-H. Kim, "Paper transistor made with covalently bonded multiwalled carbon nanotube and cellulose," Applied Physics Letters, vol. 95, no. 10, Article ID 104102, 2009.

[16] T. Sekitani, T. Yokota, U. Zschieschang et al., "Organic nonvolatile memory transistors for flexible sensor arrays," Science, vol. 326, no. 5959, pp. 1516-1519, 2009. 

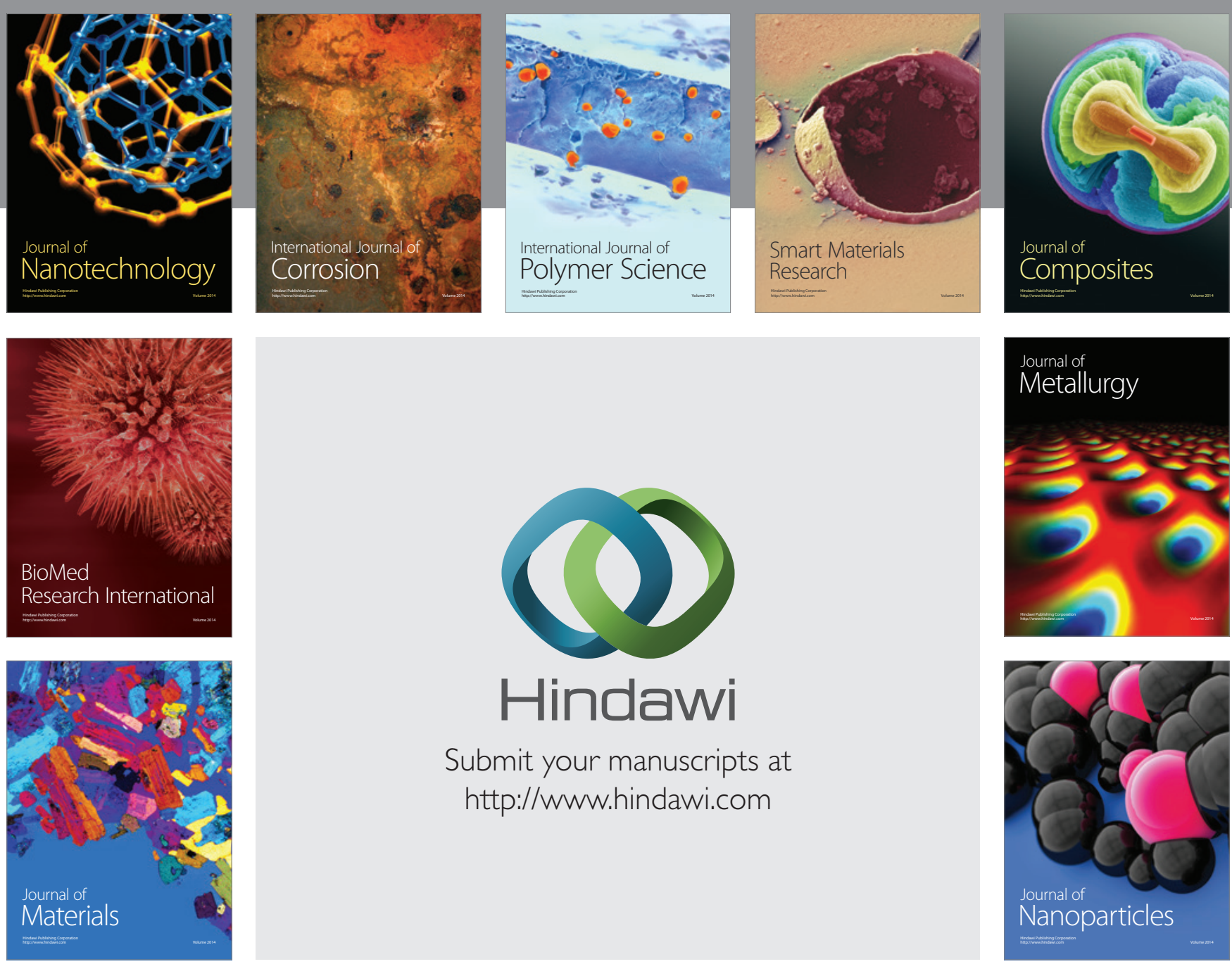

Submit your manuscripts at http://www.hindawi.com
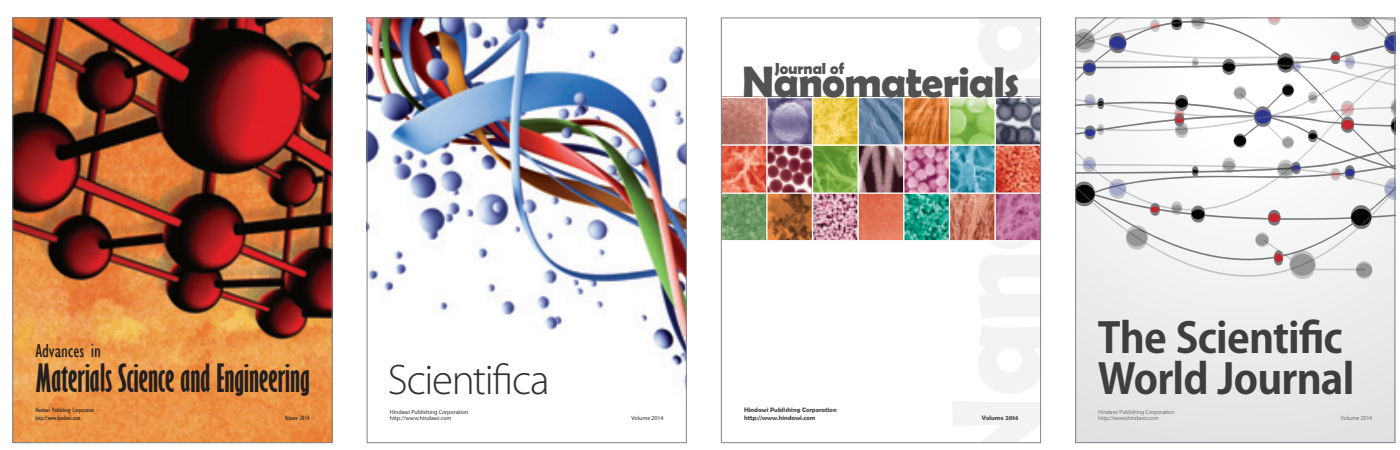

\section{The Scientific World Journal}
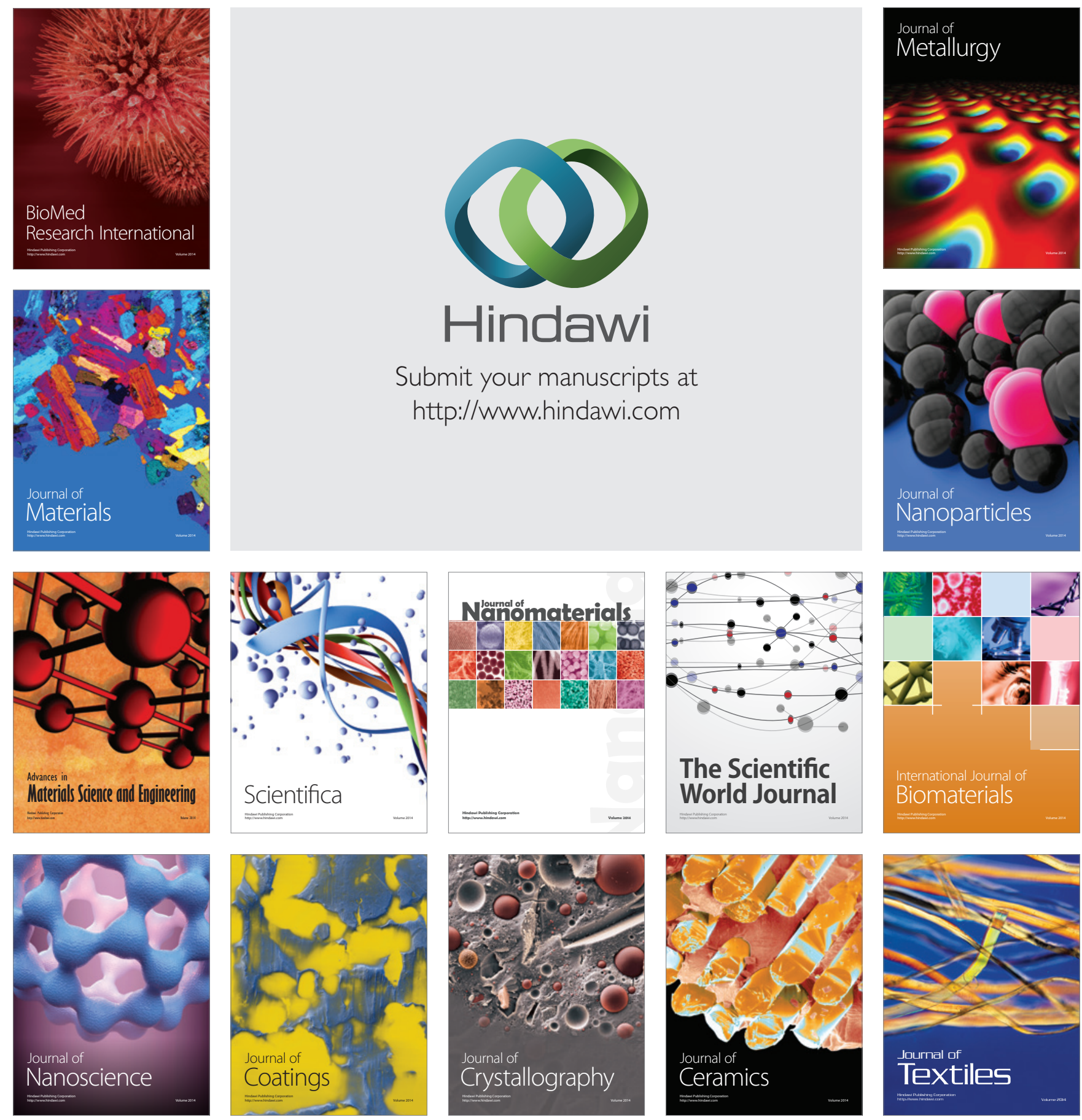